\title{
Hybrid crystalline-ITO/metal nanowire mesh transparent electrodes and their application for highly flexible perovskite solar cells
}

\author{
Hyeon-Gyun $\mathrm{Im}^{1,5}$, Seonju Jeong ${ }^{2,5}$, Jungho $\mathrm{Jin}^{3}$, Jaemin Lee ${ }^{2}$, Doo-Young Youn ${ }^{1}$, Won-Tae Koo ${ }^{1}$, \\ Sin-Bi Kang ${ }^{4}$, Hyo-Joong Kim ${ }^{4}$, Junho Jang ${ }^{1}$, Daewon Lee ${ }^{1}$, Han-Ki Kim ${ }^{4}$, Il-Doo Kim ${ }^{1}$, Jung-Yong Lee ${ }^{2}$ \\ and Byeong-Soo Bae ${ }^{1}$
}

Here, we propose crystalline indium tin oxide/metal nanowire composite electrode (c-ITO/metal NW-GFRHybrimer) films as a robust platform for flexible optoelectronic devices. A very thin c-ITO overcoating layer was introduced to the surface-embedded metal nanowire (NW) network. The c-ITO/metal NW-GFRHybrimer films exhibited outstanding mechanical flexibility, excellent optoelectrical properties and thermal/chemical robustness. Highly flexible and efficient metal halide perovskite solar cells were fabricated on the films. The devices on the c-ITO/AgNW- and c-ITO/CuNW-GFRHybrimer films exhibited power conversion efficiency values of $14.15 \%$ and $12.95 \%$, respectively. A synergetic combination of the thin c-ITO layer and the metal NW mesh transparent conducting electrode will be beneficial for use in flexible optoelectronic applications.

NPG Asia Materials (2016) 8, e282; doi:10.1038/am.2016.85; published online 24 June 2016

\section{INTRODUCTION}

Transparent conducting electrodes (TCEs) with excellent mechanical robustness will be an essential component for the next generation of flexible and stretchable optoelectronic devices, such as photovoltaic cells, light emitting devices and touch screen panels. ${ }^{1-5}$ Indium tin oxide (ITO) has unambiguously been the most widely used TCE material because of its optical transparency, thermal/chemical stability and device compatibility, coupled with its well-established fabrication processes. ${ }^{6,7}$ However, the brittle nature of ITO might limit its role in future devices. ${ }^{8}$ For instance, $150 \mathrm{~nm}$-thick amorphous-ITO (a-ITO) films or $100 \mathrm{~nm}$-thick crystalline-ITO (c-ITO) mounted on a plastic film (thickness: $100 \mu \mathrm{m}$ ) would crack with a bending radius of $<3$ mm. ${ }^{9,10}$

In addition, the poor thermal stability of optical-grade plastic films - such as polyethylene naphthalate-precludes the use of c-ITO that requires a high-temperature processing step above $250^{\circ} \mathrm{C}$ that most optical plastic films cannot stand. ${ }^{1-13}$ As such, it is not a simple task to prepare TCEs with both a high conductivity and a high mechanical flexibility using metal oxides.

Meanwhile, the metal nanowire (NW) is one of the most promising nanostructured TCE materials for the above-mentioned optoelectronic applications. ${ }^{2,14-18}$ The key merits of metal NW TCE include its excellent and tunable figure of merit (FoM), large-scale processability and, especially, its intrinsic mechanical flexibility, ${ }^{19-21}$ making this material well suited for flexible optoelectronic devices. However, the typical metal NW (Ag or Cu NW)-based TCEs also present several problematic issues, including surface roughness, weak adhesion to substrates, poor thermal/chemical stability and limited lateral conduction associated with large empty spaces between the metal NWs. ${ }^{22,23}$ In particular, the thermal/chemical instability of metal NW TCEs is the most critical drawback for this material's use in viable optoelectronic devices that require high-temperature annealing and various chemical treatments during the device fabrication. ${ }^{24,25}$

Conductive metal oxide/metal NW composite TCEs would be beneficial in terms of their thermal/chemical stability because most metal oxides are thermodynamically stable. ${ }^{26,27}$ However, two inevitable disadvantages accompany the introduction of metal oxides: (1) a degradation of the optoelectrical performance ${ }^{28,29}$ and (2) a reduction in mechanical flexibility. ${ }^{30}$ Most metal oxide/metal NW TCEs feature a deteriorated optoelectrical property, that is, the FoM, compared with that of the original metal NW TCEs; this is because most metal oxides exhibit a resistivity higher than that of metal NWs. ${ }^{1}$ Thus, thin metal

\footnotetext{
${ }^{1}$ Department of Materials Science and Engineering, Korea Advanced Institute of Science and Technology (KAIST), Daejeon, Republic of Korea; ${ }^{2}$ Graduate School of Energy, Environment, Water, and Sustainability (EEWS), Korea Advanced Institute of Science and Technology (KAIST), Daejeon, Republic of Korea; ${ }^{3}$ School of Materials Science and Engineering, University of Ulsan, Ulsan, Republic of Korea and ${ }^{4}$ Department of Advanced Materials Engineering for Information and Electronics, Kyung Hee University, Gyeonggi-do, Republic of Korea

${ }^{5}$ These authors contributed equally to this work.

Correspondence: Professor J-Y Lee, Graduate School of Energy, Environment, Water, and Sustainability (EEWS), Korea Advanced Institute of Science and Technology (KAIST), 291 Daehak-ro, Yuseong-gu, Daejeon 305-701, Republic of Korea.

E-mail: jungyong.lee@kaist.ac.kr

or Professor B-S Bae, Department of Materials Science and Engineering, Korea Advanced Institute of Science and Technology (KAIST), 291 Daehak-ro, Yuseong-gu, Daejeon 305-701, Republic of Korea.

E-mail: bsbae@kaist.ac.kr

Received 17 February 2016; revised 5 April 2016; accepted 26 April 2016
} 
oxides (such as c-ITO), which intrinsically exhibit high FoM values, would be helpful to fabricate high-performance and flexible metal oxide/metal NW composite TCEs.

Herein, we report thermally/chemically stable and highperformance c-ITO/metal NW composite TCEs on a glass fabricreinforced plastic (GFRHybrimer) film as an all-in-one TCE/substrate platform for flexible optoelectronic devices. In the composite TCE (hereafter, c-ITO/metal NW-GFRHybrimer), the thickness of the c-ITO overcoating layer was determined with the following considerations: (1) the material must retain the excellent optoelectrical and mechanical properties of the original metal NW TCEs and (2) the material must have enhanced thermal/chemical stability and lateral electrical conduction. After careful examination, we used a c-ITO overcoating layer with a thickness of $<30 \mathrm{~nm}$. Crystallization of the ITO top layer was induced directly on the metal NW-GFRHybrimer film via a $250^{\circ} \mathrm{C}$ annealing process with the aid of the excellent thermal/dimensional stability of the base film. Because very thin $(10-30 \mathrm{~nm})$ c-ITO is used as an overcoating layer, a high optical transmittance and mechanical flexibility (strain $>3 \%$ ) can be simultaneously achieved, along with an improved thermal/chemical stability and a smooth surface topography $\left(R_{\mathrm{rms}}<1 \mathrm{~nm}\right)$ that is necessary for thin film device compatibility.

In addition, we fabricated efficient, flexible perovskite solar cells with a power conversion efficiency (PCE) $>10 \%$ based on these c-ITO/metal NW-GFRHybrimer films. We also investigated the bending durability of the devices according to different bending radii $(r=5$ and $2.5 \mathrm{~mm})$. The devices were found to exhibit superior bending stability up to 500 cycles of bending.

\section{EXPERIMENTAL PROCEDURES}

\section{Synthesis of metal NW}

The AgNW solution was synthesized using a polyol process. First, $5.86 \mathrm{~g}$ of polyvinylpyrrolidone (Sigma-Aldrich, Seoul, South Korea) was dissolved in $190 \mathrm{ml}$ of glycerol (Sigma-Aldrich) and was annealed at $100^{\circ} \mathrm{C}$ for $1 \mathrm{~h}$. The solution was then cooled down to room temperature. Sodium chloride (SigmaAldrich, $0.059 \mathrm{~g})$, deionized water $(0.5 \mathrm{ml})$ and glycerol $(10 \mathrm{ml})$ were added to the solution along with silver nitrate (Sigma-Aldrich, $1.58 \mathrm{~g}$ ). The solution was heated to $160^{\circ} \mathrm{C}$ for $30 \mathrm{~min}$ and left for $1 \mathrm{~h}$. The final product was filtered using glass filters with pore sizes of $10-16 \mu \mathrm{m}$ and dispersed in methanol. The average length and diameter of the AgNWs were $11 \mu \mathrm{m}$ and $70 \mathrm{~nm}$, respectively.

The CuNW solution was synthesized using a hydrothermal process. Copper chloride (Sigma Aldrich, $0.17 \mathrm{~g}$ ) and glucose (Sigma-Aldrich, $0.1 \mathrm{~g}$ ) were dissolved in $80 \mathrm{ml}$ of distilled water. Hexadecylamine (Sigma-Aldrich, $1.44 \mathrm{~g}$ ) was added to the solution and mixed for $12 \mathrm{~h}$ with a magnetic stirrer. The solution was placed in a Teflon-lined stainless steel autoclave. Without further stirring, the solution was heated for $24 \mathrm{~h}$ at $120^{\circ} \mathrm{C}$, and a reddish brown solution was obtained. The final product was filtered and dispersed in methanol. The average length and diameter of the CuNWs were $35 \mu \mathrm{m}$ and $50 \mathrm{~nm}$, respectively.

\section{Fabrication of metal NW-GFRHybrimer films}

The metal NW-GFRHybrimer films were fabricated by transferring the metal NW networks, which are preformed on a donor glass, onto the surface of a base GFRHybrimer film via a vacuum-bag molding process. The metal NW networks on the donor glass substrates are prepared by using an automated spray coater. The back pressure $\left(\mathrm{N}_{2}\right)$ was $0.1 \mathrm{MPa}$, and the flow rate of the metal NW solution was $3 \mathrm{ml} \mathrm{min}^{-1}$. The nozzle-to-substrate distance was $12 \mathrm{~cm}$, and the nozzle scan speed was 4000 inch $\mathrm{min}^{-1}$. The temperature of the donor glass substrate was kept at $100^{\circ} \mathrm{C}$ for immediate solvent evaporation, and the humidity in the spraying booth was maintained at $30 \%$. For the CuNWs, the preformed CuNW network on the donor glass was annealed using a tube furnace under a $\mathrm{H}_{2}$ atmosphere for $1 \mathrm{~h}$ at $200{ }^{\circ} \mathrm{C}$ to eliminate residual surface oxides that formed after spray coating. The preformed metal NW network on the first donor glass was brought into contact with a glass-fabric cloth impregnated with a matrix resin on the second donor glass, and the assembly was compressed. This was followed by vacuum bag molding and ultraviolet curing. The matrix was a transparent resin blend consisting of cycloaliphatic epoxy oligosiloxanes (CAEO) and bis-[1-ethyl(3-oxetanyl)] methyl ether (DOX) as a functional crosslinker. Finally, the separation of the two donor glasses resulted in the transparent and freestanding metal NW-GFRHybrimer film of which the thickness was ca. $60 \mu \mathrm{m}$.

\section{ITO layer formation}

The ITO layer was deposited using DC magnetron sputtering with a working pressure of 2 mTorr, a gas flow rate of $\mathrm{Ar} / \mathrm{O}_{2}$ of 20/0.3 SCCM and a DC power of $100 \mathrm{~W}$ at room temperature. The as-deposited a-ITO layer was then annealed at $250^{\circ} \mathrm{C}$ under a $\mathrm{N}_{2}$ atmosphere.

\section{Transparent electrode characterization}

The sheet resistance $\left(R_{\mathrm{sh}}\right)$ was measured using a 4-point probe sheet resistance meter. The total optical transmittance was evaluated using a ultraviolet-visible spectrometer under the GFRHybrimer film baseline conditions. The scanning electron microscope (s4800, HITACHI, Tokyo, Japan) and transmission electron microscope (Titan cubed G2 60-300, FEI, Hillsboro, OR, USA) images were obtained. X-ray diffraction analysis was conducted using a multipurpose high-power X-ray diffractometer (D/Max-2500, Rigaku, Tokyo, Japan). An atomic force microscopy analysis was conducted using a scanning probe microscope (XE-100, Park Systems, Suwon, South Korea). For mechanical, thermal and chemical stability tests, c-ITO/AgNW-GFRHybrimer $\left(R_{\mathrm{sh}}=37 \Omega \mathrm{sq}^{-1}\right)$ and c-ITO/CuNW-GFRHybrimer $\left(R_{\mathrm{sh}}=42 \Omega \mathrm{sq}^{-1}\right)$, which have a $10 \mathrm{~nm}$ top ITO layer, were used.

\section{Device fabrication and characterization}

Perovskite solar cells were fabricated on various TCE substrates including c-ITO/AgNW- or CuNW-GFRHybrimer, ITO/glass and ITO/polyethylene terephthalate (PET) with a device structure of substrate/PEDOT:PSS $(40 \mathrm{~nm}) /$ perovskite $(300 \mathrm{~nm}) / \mathrm{PC} 60 \mathrm{BM}(40 \mathrm{~nm}) / \mathrm{BCP}(8 \mathrm{~nm}) / \mathrm{Ag}(150 \mathrm{~nm})$. Poly(3,4-ethylenedioxythiophene)-poly(styrenesulfonate) (PEDOT/PSS, Baytron (Goslar, Germany) polyvinylpyrrolidone $\mathrm{Al} 4083$ ) was spun onto a cleaned TCE substrate at 4000 r.p.m. for $30 \mathrm{~s}$ and dried at $140{ }^{\circ} \mathrm{C}$ for $15 \mathrm{~min}$. The perovskite solution was prepared by dissolving $\mathrm{CH}_{3} \mathrm{NH}_{3} \mathrm{I}$ (1-material) and $\mathrm{PbI}_{2}$ (99\%, Aldrich) with a molar ratio of 1:1 in a mixture of $\gamma$-butyrolactone/ dimethyl sulfoxide $(7: 3 \mathrm{v} / \mathrm{v})$ at $70{ }^{\circ} \mathrm{C}$ for $12 \mathrm{~h}$. The solution was spun onto the PEDOT/PSS layer by a consecutive three-step processes at 500, 1000 and $2000 \mathrm{r}$. p.m. for 5, 10 and $30 \mathrm{~s}$, respectively. During the third step, the perovskite layer was treated with a toluene droplet $(\sim 70 \mu \mathrm{l})$, and then immediately dried on a hot plate at $100^{\circ} \mathrm{C}$ for $10 \mathrm{~min}$. The [6,6]-Phenyl-C61-butyric acid methyl ester $\left(\mathrm{PC}_{60} \mathrm{BM}\right)$ in chlorobenzene ( $\left.1 \mathrm{wt} \%\right)$ was spun on top of the perovskite layer at 3000 r.p.m. for 45 s. A BCP layer $(8 \mathrm{~nm})$ and an Ag layer $(150 \mathrm{~nm})$ were sequentially deposited through a shadow mask by thermal evaporation at $10^{-7}$ Torr. The current density-voltage $(\mathrm{J}-\mathrm{V})$ characteristics were measured under an irradiance of $100 \mathrm{~mW} \mathrm{~cm}^{-2}$ from a $150 \mathrm{~W}$ xenon short-arc lamp filtered by an air mass $1.5 \mathrm{G}$ filter with a K201 LAB55 solar simulator (PEC-L12, Peccell Technologies McScience, Seoul, Korea).

\section{RESULTS AND DISCUSSION}

\section{Fabrication of c-ITO/metal NW-GFRHybrimer films}

Figure 1a schematically illustrates the fabrication process of a c-ITO/metal NW-GFRHybrimer film. ${ }^{31}$ Briefly, the metal NWGFRHybrimer films (AgNW- and CuNW-GFRHybrimer) are first fabricated by transferring a metal NW network (AgNW or CuNW) onto the surface of a base GFRHybrimer film; then, a thin a-ITO (thickness of $10-30 \mathrm{~nm}$ ) is sputtered on the film. Subsequently, the films are annealed at $250^{\circ} \mathrm{C}$ under a $\mathrm{N}_{2}$ atmosphere to induce the thermally driven crystallization of the ITO layer. It is worthwhile to note that the top ITO layer is directly crystallized on the flexible GFRHybrimer film; such a crystallization is not commonly achievable 
a
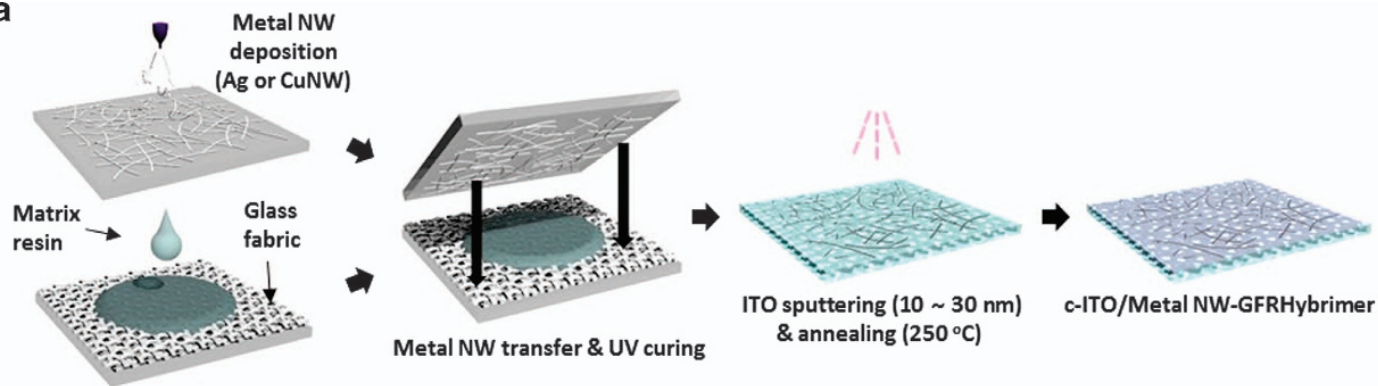

ITO sputtering (10 $30 \mathrm{~nm})$

c-ITO/Metal NW-GFRHybrimer film

Metal NW transfer \& UV curing \& annealing $\left(250^{\circ} \mathrm{C}\right)$
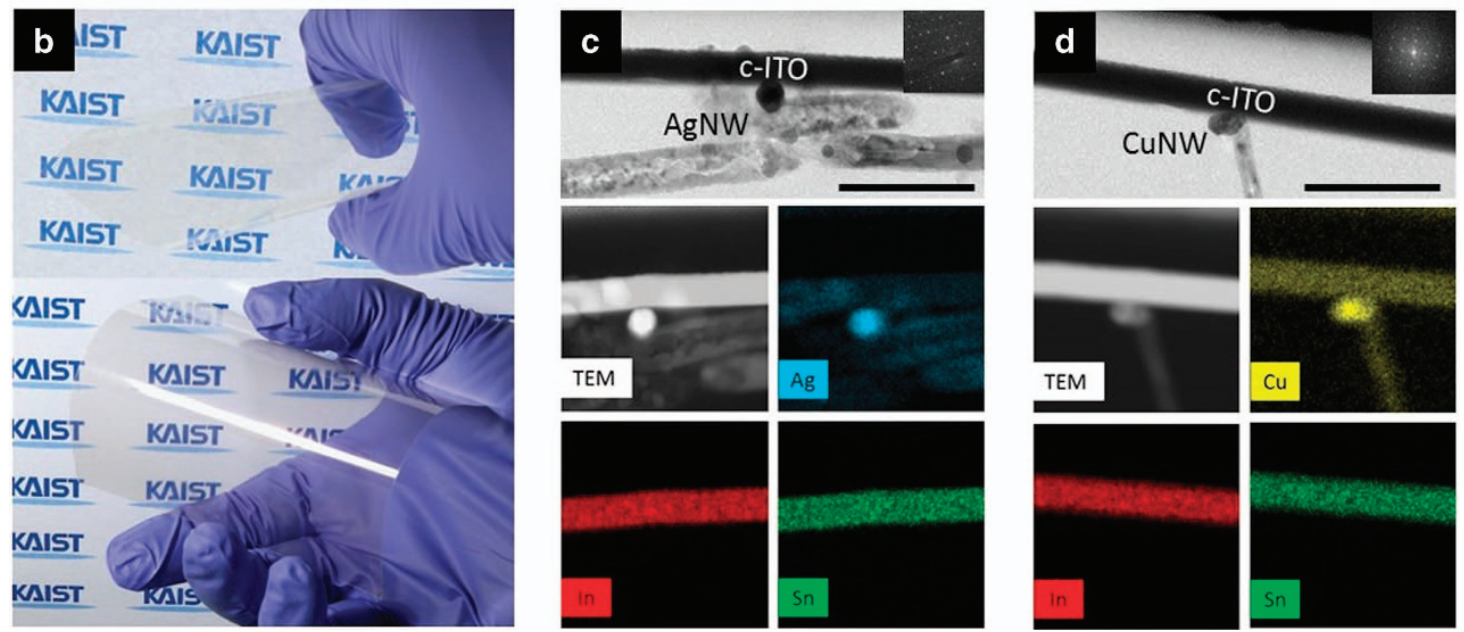

Figure 1 (a) Fabrication procedure for the c-ITO/metal NW-GFRHybrimer films. (b) Photographs of the c-ITO/AgNW- (top) and c-ITO/CuNW-GFRHybrimer films (down). (c, d) Transmission electron microscopy (TEM) and energy-dispersive spectroscopy (EDS) elemental mapping images of the (c) c-ITO/AgNW- and (d) C-ITO/CuNW-GFRHybrimer films. The insets are diffraction patterns of the crystallized ITO top layers. The scale bars: $100 \mathrm{~nm}$. AgNW, silver nanowire; c-ITO, crystalline indium tin oxide; CuNW, copper nanowire; NW, nanowire.

on typical plastic films, such as polyethylene naphthalate, that have low glass transition temperatures. ${ }^{10}$ Figure $1 \mathrm{~b}$ shows the transparent c-ITO/metal NW-GFRHybrimer films of which the sizes are $10 \times 10 \mathrm{~cm}^{2}$.

Transmission electron microscopy analyses of the c-ITO/metal NW electrode revealed direct contact between the bottom-embedded metal NW network and the top c-ITO (Figures $1 \mathrm{c}$ and d). This was further evaluated by X-ray energy-dispersive spectroscopy elemental mapping. It was found that the indium and tin distributions are clearly separated from the silver or copper distribution. Surface and tilted scanning electron microscope images were also used to visualize the hybrid structure of the c-ITO/metal NW-GFRHybrimer films (Supplementary Figure S1). By introducing the c-ITO top layer, an exceptionally smooth surface topography for the c-ITO/metal NW-GFRHybrimer films $\left(R_{\mathrm{rms}}<1 \mathrm{~nm}\right.$ and maximum peak-to-peak value: $\sim 5 \mathrm{~nm}$ ) was attained, as seen in the topographic atomic force microscopy images (Supplementary Figure S2).

To verify the thermally induced crystallization of the top ITO layer, $\mathrm{X}$-ray diffraction analyses of the a-ITO/AgNW- and a-ITO/CuNWGFRHybrimers were conducted before/after annealing at $250{ }^{\circ} \mathrm{C}$ under a $\mathrm{N}_{2}$ atmosphere (Figure 2a). Before annealing, the amorphous phase of ITO was observed, along with characteristic peaks only from silver or copper. ${ }^{32,33}$ After 4 h of annealing, however, the characteristic peaks of c-ITO $\left(2 \theta=30.5^{\circ}\right.$ and $2 \theta=35.5^{\circ}$ assigned to 222 and 400 , respectively) were observed. ${ }^{10,34}$ These peaks imply that the top ITO layer was successfully crystallized by the annealing process.

To evaluate the effect of the ITO layer on the electrical performance, the sheet resistance values $\left(R_{\mathrm{sh}}\right)$ of various films (metal NW-, a-ITO- and a-ITO/metal NW-GFRHybrimers) were measured as a function of the annealing time and by varying the ITO thickness (Figure $2 \mathrm{~b}$ and Supplementary Figure S3). For example, after an a-ITO deposition $(30 \mathrm{~nm})$ on the AgNW-GFRHybrimer film, the $R_{\text {sh }}$ value was $45 \Omega \mathrm{sq}^{-1}$. After $2 \mathrm{~h}$ of annealing, the $R_{\mathrm{sh}}$ values of the a-ITO/metal NW-GFRHybrimer films were noticeably decreased; these values saturated at $\sim 17 \Omega \mathrm{sq}^{-1}$ with a prolonged annealing time of $4 \mathrm{~h}$. This indicates that the thermally induced crystallization of the ITO layer resulted in an improved electrical conduction. Indeed, the $R_{\mathrm{sh}}$ value $\left(17.2 \Omega \mathrm{sq}^{-1}\right)$ of the hybrid electrodes was less than that of parallel-connected ITO $\left(162 \Omega \mathrm{sq}^{-1}\right)$ or that of a NW network (81 $\Omega \mathrm{sq}^{-1}$ ), implying that AgNWs were further welded during the ITO crystallization.

Introducing a metal oxide to a metal NW leads to the optoelectrical performance degradation of the TCE because of the resistivity of the metal oxides that is higher than that of the metal NWs. ${ }^{28,29}$ To determine the effect of the c-ITO layer on the optoelectrical performance, the total transmittance at $550 \mathrm{~nm}\left(T_{\text {tot }}\right)$ of the c-ITO/ metal NW-GFRHybrimer films with varying ITO thicknesses (10, 20 and $30 \mathrm{~nm}$ ) was measured with respect to the bare GFRHybrimer. The $T_{\text {tot }}$ vs $R_{\text {sh }}$ for the various TCEs (metal NW-, c-ITO- and c-ITO/metal NW-GFRHybrimers) are plotted (Figure $2 \mathrm{c}$ ); reference $\sigma_{\mathrm{DC}} / \sigma_{\mathrm{op}}$ curves are also included. The following equation provides the FoM for the nanostructured thin film TCEs: ${ }^{35}$

$$
T=\left(1+\frac{188.5}{R_{\mathrm{sh}}} \frac{\sigma_{\mathrm{op}}}{\sigma_{\mathrm{DC}}}\right)
$$

where $\sigma_{\mathrm{DC}}$ is the DC conductivity and $\sigma_{\mathrm{op}}$ is the optical conductivity of 
a

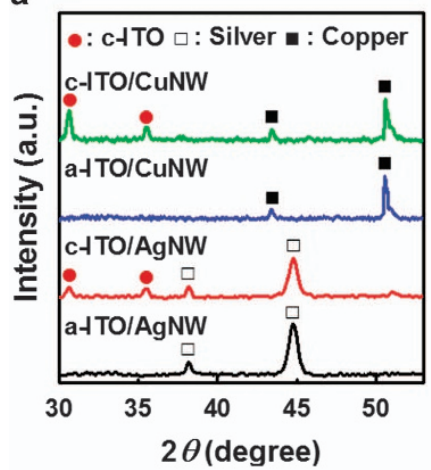

d

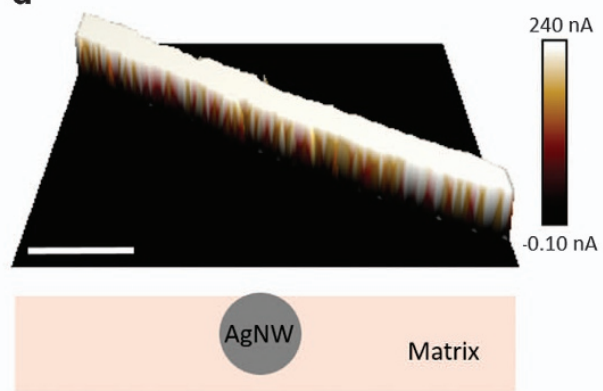

b

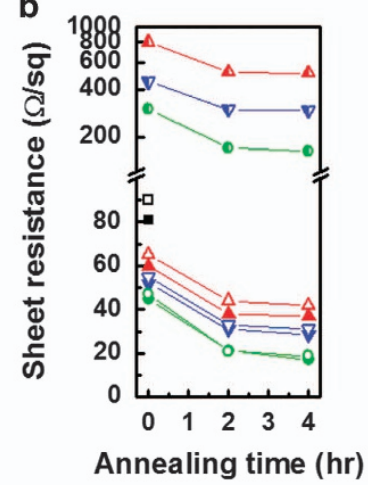

c

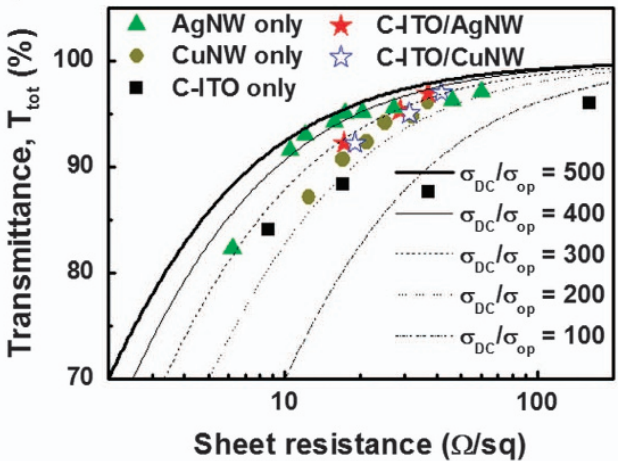

e

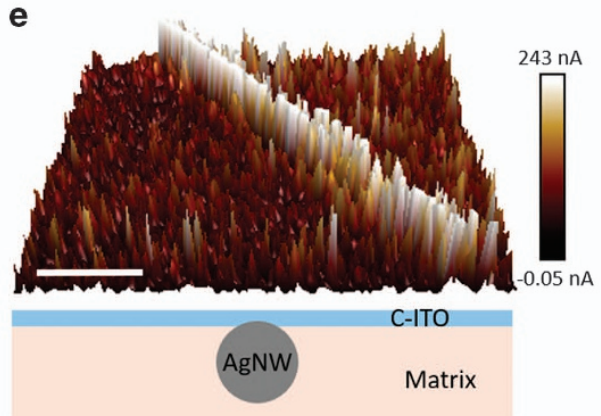

Figure 2 (a) X-ray diffraction (XRD) patterns of the ITO/metal NW-GFRHybrimer films. (b) A plot of sheet resistance ( $R_{\text {sh }}$ ) vs annealing time for the ITO-only (half-filled), ITO/AgNW-GFRHybrimer (filled) and ITO/CuNW-GFRHybrimer (voided) films with various ITO thicknesses. Triangles, reverse triangles and circles indicate an ITO thickness of 10, 20 and $30 \mathrm{~nm}$, respectively; filled and voided squares indicate the bare AgNW- and CuNW-GFRHybrimer films, respectively. Refer to Supplementary Information for more detail. (c) The total transmittance ( $T_{\text {tot }}$ ) vs $R_{\text {sh }}$ for various transparent electrodes. (d, e) Conductive atomic force microscopy (c-AFM) scan images, with current mappings for the (d) AgNW-GFRHybrimer and the (e) c-ITO/AgNW-GFRHybrimer. The scale bar, 400 nm (bottom images are schematic illustrations of the transparent conducting electrodes (TCEs)). AgNW, silver nanowire; c-ITO, crystalline indium tin oxide; CuNW, copper nanowire; NW, nanowire.

the film. $\sigma_{\mathrm{DC}} / \sigma_{\mathrm{op}}$ is defined as the FoM. As seen in Figure $2 \mathrm{c}$, the value of $\sigma_{\mathrm{DC}} / \sigma_{\mathrm{op}}$ of $\mathrm{c}-\mathrm{ITO}$ was $<300$, and the $\sigma_{\mathrm{DC}} / \sigma_{\mathrm{op}}$ of the AgNW TCE was 500 . After introducing the c-ITO layer, the $\sigma_{\mathrm{DC}} / \sigma_{\mathrm{op}}$ of the c-ITO/ AgNW TCE becomes 300. This illustrates that use of the c-ITO/AgNW composite TCE results in a slightly degraded optoelectrical performance compared with the case of the AgNW TCE. For the CuNW TCE, the optoelectrical performance was retained after introducing the c-ITO layer $\left(\sigma_{\mathrm{DC}} / \sigma_{\mathrm{op}}\right.$ of 300$)$. These results suggest that the optoelectrical performance degradation of metal oxide/metal NW TCEs can be minimized if the FoM difference between the metal oxide and the metal NW is small. Our c-ITO/metal NW-GFRHybrimer films exhibit an excellent optoelectrical performance compared with those of the state-of-the-art metal NW composite TCEs; ${ }^{26,29,36}$ for example, values of $R_{\mathrm{sh}}=17.2 \Omega \mathrm{sq}^{-1}$ at $T_{\mathrm{tot}}=92.3 \%$ for c-ITO/AgNW-GFRHybrimer and $R_{\mathrm{sh}}=19 \Omega \mathrm{sq}^{-1}$ at $T_{\text {tot }}=92.2 \%$ for c-ITO/CuNW-GFRHybrimer were achieved (Table 1).

The introduction of a metal oxide layer, by filling the voids between the metal NW networks, can help inducing additional charge for carrier collection/injection. ${ }^{37}$ To evaluate the lateral conduction behavior, conductive atomic force microscopy was performed on the c-ITO $(30 \mathrm{~nm}) / \mathrm{AgNW}$-GFRHybrimer films. For the bare AgNWGFRHybrimer film, the carrier conduction was observed only through the embedded metal NW networks (Figure 2d). In contrast, for the c-ITO/AgNW-GFRHybrimer sample, the current flow was detected in regions where no metal NWs were located (Figure 2e). Though carrier conduction mainly occurs through the metal NW network, lateral conduction (an average $20 \%$ of the current on the NWs) was also observed where c-ITO was deposited on the voids.
Mechanical, thermal and chemical robustness of metal oxide/metal NW hybrid TCEs

The mechanical robustness of the metal oxide/metal NW TCEs was analyzed to confirm the applicability of this material to flexible devices. To evaluate the durability of the c-ITO/metal NWGFRHybrimer films at different bending radii $(r)$, a series of bending tests for different c-ITO thicknesses were performed with various $r$ (Figure 3a). The 10 and $20 \mathrm{~nm}$ c-ITO/metal NW-GFRHybrimer films (thickness: $60 \mu \mathrm{m}$ ) showed little change in the value of $R_{\mathrm{sh}}$ until $r$ reached $1 \mathrm{~mm}$ (strain of $3 \%$ ). For the $30 \mathrm{~nm} \mathrm{c-ITO/metal} \mathrm{NW-}$ GFRHybrimer films, the value of $R_{\mathrm{sh}}$ began to increase when $r=1.5 \mathrm{~mm}$ (strain of $2 \%$ ). In contrast, the commercial ITO/PET sample $(150 \mathrm{~nm}$ of ITO on $200 \mu \mathrm{m}$ of PET) started to crack at $r=5.5 \mathrm{~mm}$ (strain of $1.8 \%$ ). These results indicate that the mechanical durability of the metal oxide/metal NW TCEs is limited by the thickness of the metal oxide layer, and a c-ITO layer thickness of $<20 \mathrm{~nm}$ is necessary for the flexibility of the hybrid TCE.

To confirm the repeated bending durability, the change in the resistance of the $10 \mathrm{~nm} \mathrm{c-ITO/metal} \mathrm{NW-GFRHybrimer} \mathrm{films} \mathrm{with}$ cyclic bending ( $r=1 \mathrm{~mm}$ ) was also monitored (Figure $3 \mathrm{~b}$ ). The c-ITO/ metal NW-GFRHybrimer films tolerate $10^{4}$ bending cycles without any sign of mechanical failure, retaining their initial $R_{\mathrm{sh}}$ values. In sharp contrast, the $R_{\mathrm{sh}}$ value of the reference ITO/PET film catastrophically increased 28 times after 10 cycles of the bending test. The repeated bending durability was further confirmed by surface scanning electron microscope analysis (Supplementary Figure S4). In the case of the c-ITO/metal NW-GFRHybrimer films, no cracks formed after the repeated bending test. However, large cracks were observed on 
Table $1 \boldsymbol{R}_{\mathrm{sh}}$, total transmittance and figure of merit (FoM) of the c-ITO/metal NW-GFRHybrimer films with various ITO thicknesses

\begin{tabular}{lll}
\hline ITO thickness $(\mathrm{nm})$ & $\mathrm{R}_{\text {sh }}\left(\Omega \mathrm{sq} \mathrm{C}^{-1}\right)$ & $\mathrm{T}$ at $550 \mathrm{~nm}$ (\%) \\
\hline $\begin{array}{l}\text { c-ITO/AgNW } \\
0\end{array}$ & 81 & 97.2 \\
10 & 37 & 96.9 \\
20 & 28.6 & 95.3 \\
30 & 17.2 & 92.3 \\
& & \\
C-ITO/CuNW & & \\
0 & 90 & 97.4 \\
10 & 41.9 & 97 \\
20 & 31.2 & 95 \\
30 & 19 & 92.2 \\
\hline
\end{tabular}

Abbreviations: AgNW, silver nanowire; c-ITO, crystalline indium tin oxide; CuNW, copper nanowire; $R_{\mathrm{sh}}$, sheet resistance.

the reference ITO/PET film after 10 cycles of bending. This result highlights that the mechanically robust c-ITO/metal NW-GFRHybrimer films can be suitable for typical flexible optoelectronic applications because the performance degradation of flexible devices upon repeated mechanical bending is mostly associated with the failure of the TCE layer that shows a drastic increase in $R_{\mathrm{sh}}{ }^{13}$

The thermal-oxidation stability of the metal NW TCEs should be guaranteed for this material to be suitable for fabricating optoelectronic devices, such as perovskite solar cells. To evaluate the thermaloxidation stability, the c-ITO/metal NW-GFRHybrimer films were oven annealed under the ambient atmosphere; $R_{\mathrm{sh}}$ values were measured during annealing. The annealing temperatures were $300{ }^{\circ} \mathrm{C}$ and $100^{\circ} \mathrm{C}$ for the c-ITO/AgNW- and c-ITO/CuNW-GFRHybrimers, respectively. The lower temperature for the c-ITO/CuNW-GFRHybrimer was applied because copper is more prone to oxidation than silver is. ${ }^{38}$ The AgNW on glass substrate shows an immediate degradation of the electrical performance (Figure 3c). Although the degradation time of the AgNW-GFRHybrimer film is prolonged compared with that of the AgNW on glass, the AgNWGFRHybrimer film also shows a dramatic increase in the value of $R_{\mathrm{sh}}$ after $150 \mathrm{~min}$ of annealing. In contrast, the value of $R_{\mathrm{sh}}$ of the c-ITO/AgNW-GFRHybrimer film was maintained after $400 \mathrm{~min}$ of annealing. A similar tendency was also observed for the CuNW TCEs (Figure 3d). Contrary to the cases of CuNW on glass and of the CuNW-GFRHybrimer film, the c-ITO/CuNW-GFRHybrimer film was found to retain its electrical performance even after $240 \mathrm{~h}$ of annealing. These results indicate that a bottom-embedded metal NW network is effectively protected from oxidation by a top ITO overcoating layer. ${ }^{24,26,39}$

The chemical stability of the metal NW TCEs is another important issue because many device fabrication processes involve chemicals that could degrade the bottom metal NW network. To determine the chemical stability of c-ITO/metal NW-GFRHybrimer films, $\mathrm{K}_{2} \mathrm{~S}$ corrosion tests were performed. A $\mathrm{K}_{2} \mathrm{~S}$ aqueous solution (5 wt\%, $2 \mathrm{ml}$ ) was used as an oxidative reagent because sulfur is corrosive to AgNWs and CuNWs. The $R_{\text {sh }}$ values of each sample were in situ measured through two electrical outlets formed with silver paste while the $\mathrm{K}_{2} \mathrm{~S}$ solution was spread on the samples. Upon exposure to the $\mathrm{K}_{2} \mathrm{~S}$ solution, the $R_{\text {sh }}$ values of the AgNW on glass substrate and the AgNW-GFRHybrimer film increased abruptly within 60 and $200 \mathrm{~s}$, respectively (Figure $3 \mathrm{e}$ ). In contrast, the electrical performance of the c-ITO/AgNW-GFRHybrimer film was stable even after $1000 \mathrm{~s}$, confirming the superior chemical stability of this material. In a similar manner, the c-ITO/CuNW-GFRHybrimer film also retained its electrical performance for $300 \mathrm{~s}$ of the same test (Figure $3 \mathrm{f}$ ). However, the $R_{\mathrm{sh}}$ value of the CuNW on the glass sample surged immediately, and the CuNW-GFRHybrimer film also showed a drastic increase in the value of $R_{\mathrm{sh}}$ after $40 \mathrm{~s}$. This improved chemical stability of the c-ITO/metal NW-GFRHybrimer films can also be attributed to the top ITO overcoating layer that effectively protects the bottom metal NW network from chemical reagents. The excellent thermal-oxidation/ chemical stability of the c-ITO/metal NW-GFRHybrimer films means that this material can provide an excellent platform for fabricating optoelectronic devices.

\section{Organic/inorganic perovskite solar cells on c-ITO/metal NW-GFRHybrimer films}

To demonstrate the potential of the c-ITO/metal NW-GFRHybrimer films as a flexible TCE/substrate platform for optoelectronic devices, organic/inorganic perovskite solar cells using methyl ammonium lead iodide $\left(\mathrm{CH}_{3} \mathrm{NH}_{3} \mathrm{PbI}_{3}\right)$ were fabricated on the c-ITO/metal NWGFRHybrimer films. It is challenging to fabricate perovskite solar cells directly on metal NW TCEs because of the high reactivity of the metal NWs toward halides. ${ }^{40}$ The device structure is c-ITO/metal NW-GFRHybrimer/PEDOT:PSS/perovskite/PCBM/BCP/Ag; a crosssectional transmission electron microscopy image is shown in Figures $4 \mathrm{a}$ and $\mathrm{b}$.

Figure $4 \mathrm{c}$ shows the representative current density-voltage $(\mathrm{J}-\mathrm{V})$ characteristics of the perovskite solar cell devices on c-ITO/AgNWGFRHybrimer, c-ITO/CuNW-GFRHybrimer, ITO/glass and ITO/PET under $100 \mathrm{~mW} \mathrm{~cm}^{-2}$ AM $1.5 \mathrm{G}$ illumination. The reference devices on the ITO/glass and ITO/PET show PCE values of $15.38 \%$ and $12.08 \%$, respectively. The devices on the c-ITO/AgNW- and c-ITO/ CuNW-GFRHybrimer films exhibit PCE values of $14.15 \%$ and $12.95 \%$, respectively. The photovoltaic performance of the perovskite solar cells is summarized in Table 2. It should be noted that the performance of the devices on the c-ITO/metal NW-GFRHybrimer films are comparable to or even superior to those of the reference devices. It is worth noting that the excellent chemical stability of the c-ITO/metal NW-GFRHybrimer films against the perovskite precursor solution enables stable integration of the perovskite layer (Supplementary Figure S5).

The $V_{\mathrm{oc}}$ values were improved in the devices on both the c-ITO/ AgNW- and c-ITO/CuNW-GFRHybrimer films compared with those values of the reference ITO-based devices. These higher values of $V_{\mathrm{oc}}$ can be attributed to the higher work function of the TCEs (Supplementary Figure S6) that could induce a larger built-in potential $\left(V_{\mathrm{bi}}\right)^{41}$

On the other hand, c-ITO/metal NW-GFRHybrimer-based devices show lower $J_{\text {sc }}$ and $F F$ values compared with those of the control devices. To investigate the origin of the lower $J_{\text {sc }}$ values, we compared the transmittance of the electrodes and the absorption of the devices (Supplementary Figure S7). The c-ITO/AgNW-GFRHybrimer and the c-ITO/CuNW-GFRHybrimer exhibited lower transmittance values than the ITO electrode below 500 and $600 \mathrm{~nm}$, respectively; these results were partly because of the surface plasmon absorption of AgNWs and CuNWs. This result was also confirmed in the external quantum efficiency spectra of the devices (Supplementary Table S1 and Supplementary Figure S8). ${ }^{42,43}$ Furthermore, the low FF values of the c-ITO/metal NW-GFRHybrimer devices are attributable to the higher series resistance that is due to the leakage current, resulting from the discontinuous metal NW networks that form when $10 \mathrm{~nm}$ of thin ITO is deposited on the metal NW-GFRHybrimer. To evaluate the reliability of the perovskite solar cell devices on c-ITO/ 
a

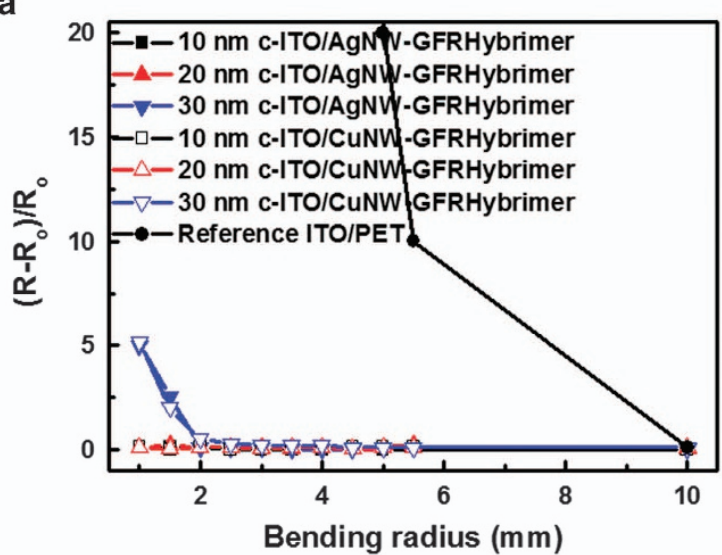

C

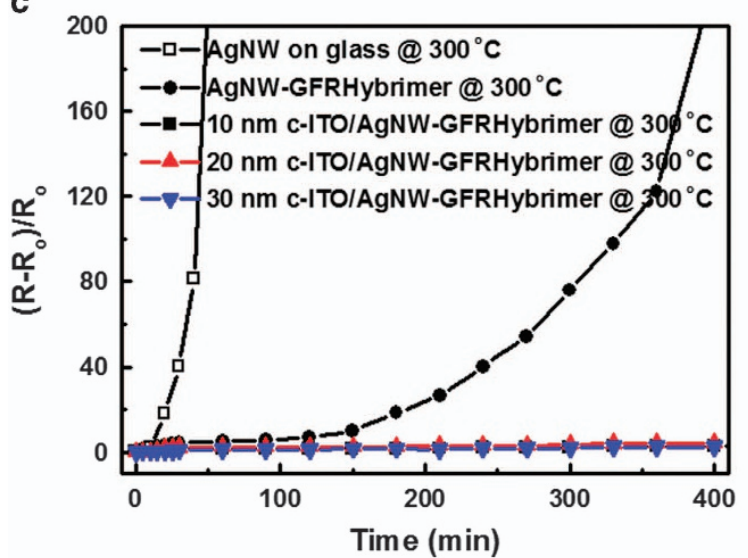

e

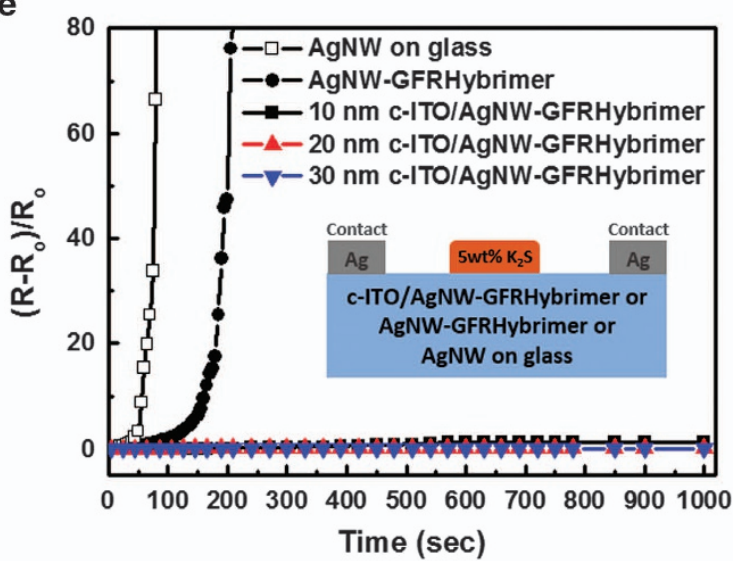

b

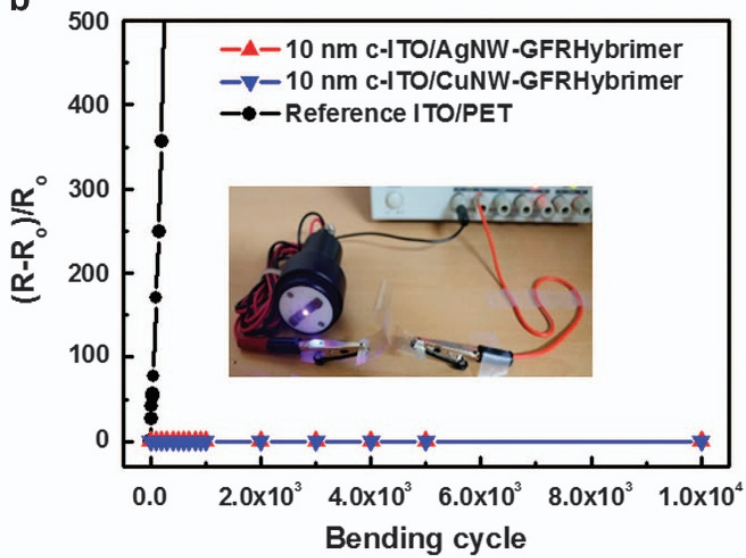

d

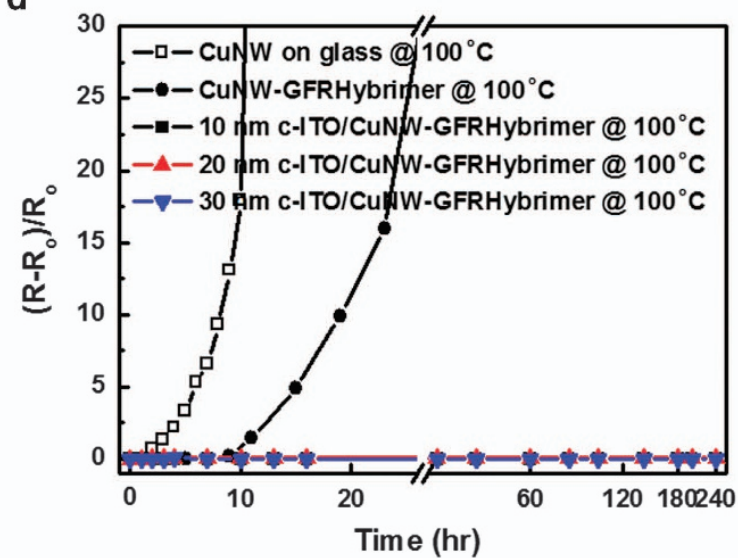

f

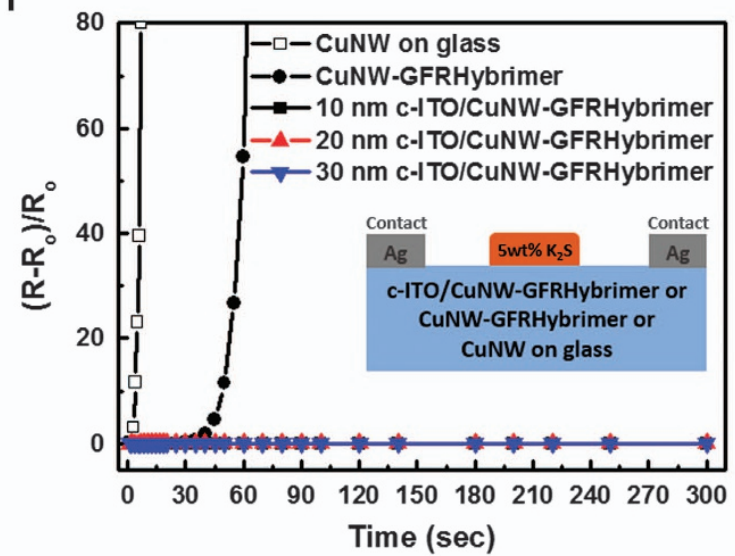

Figure 3 Mechanical, thermal and chemical stabilities of the c-ITO/metal NW-GFRHybrimer films. (a) The bending test of various films with different c-ITO thicknesses and bending radii. (b) The cyclic bending test of the $10 \mathrm{~nm}$ c-ITO/metal NW-GFRHybrimer films; the bending radius is $1 \mathrm{~mm}$. The inset represents a bent c-ITO/AgNW-GFRHybrimer film connected in an electrical circuit with a power supply and an LED. (c, d) The resistance changes of the (c) C-ITO/AgNW-GFRHybrimer annealed at $300^{\circ} \mathrm{C}$ and of the (d) c-ITO/CuNW-GFRHybrimer annealed at $100{ }^{\circ} \mathrm{C}$. (e, f) The $\mathrm{K}_{2} \mathrm{~S}$ corrosion test for the (e) C-ITO/ AgNW- and (f) C-ITO/CuNW-GFRHybrimer films. AgNW, silver nanowire; c-ITO, crystalline indium tin oxide; CuNW, copper nanowire; NW, nanowire.

AgNW-GFRHybrimer, c-ITO/CuNW-GFRHybrimer and ITO/glass, statistical data for the PCEs of 20 devices are presented in Supplementary Figure S9.

Next, to verify the mechanical durability of the perovskite solar cells on c-ITO/metal NW-GFRHybrimer films, a bending test was performed with different bending radii for multiple cycles (Figure $4 \mathrm{~d}$ ). The photovoltaic performances of the devices on the c-ITO/metal
NW-GFRHybrimer films and on ITO/PET were measured after repeated bending cycles with bending radii of 2.5 and $5 \mathrm{~mm}$. Nearly the same photovoltaic performance was observed for the devices on the c-ITO/metal NW-GFRHybrimer films after 500 cycles of bending. Although the $J_{\mathrm{sc}}$ and $F F$ values of the devices were slightly degraded, possibly because of the degradation of the perovskite layer, the PCEs of the devices on c-ITO/AgNW- and c-ITO/CuNW-GFRHybrimer films 
a

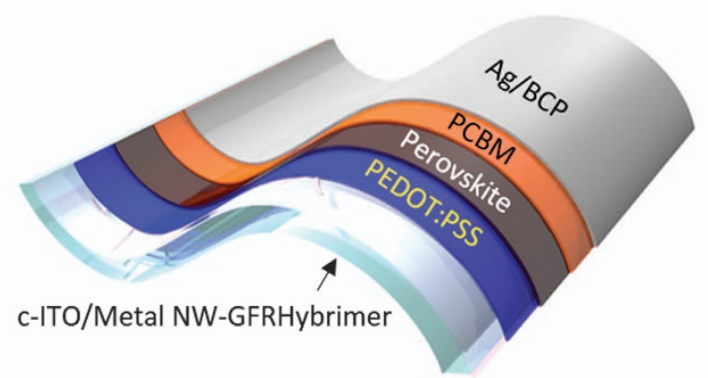

C

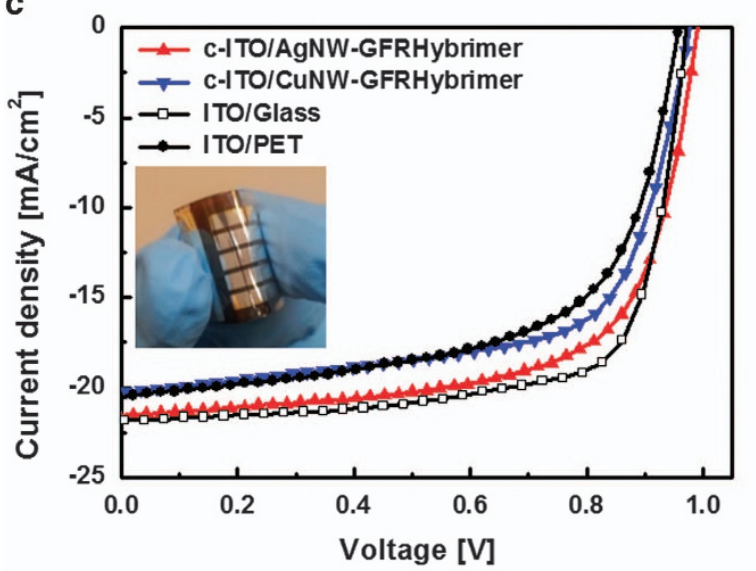

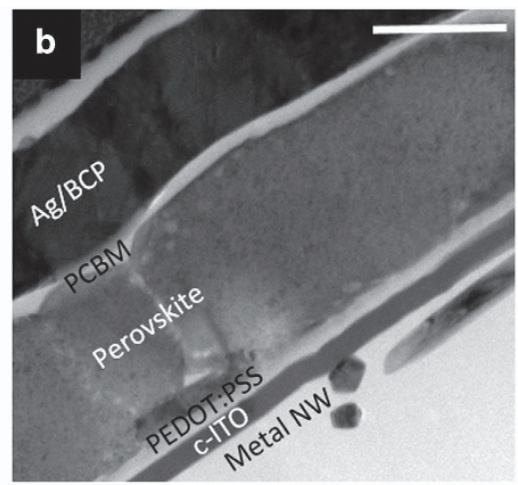

d

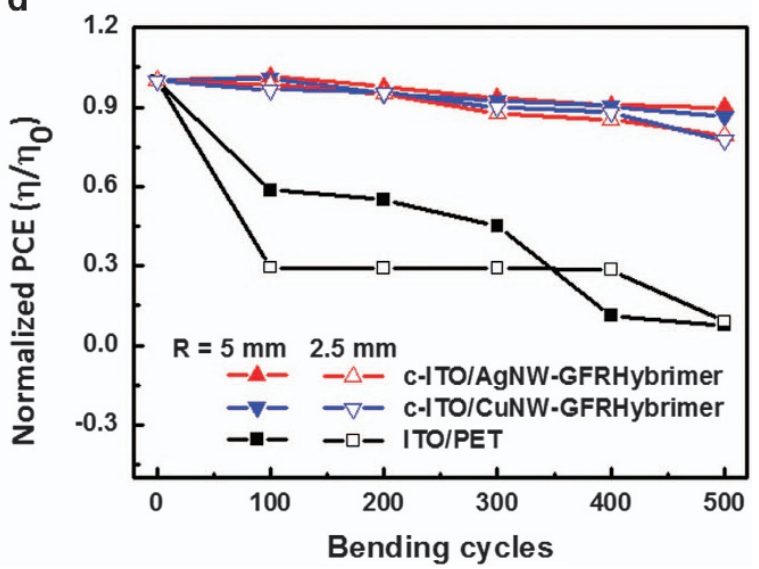

Figure 4 Flexible perovskite solar cell device on the c-ITO/Metal NW-GFRHybrimer films. (a) A schematic illustration of the device structure. (b) The transmission electron microscopy (TEM) image of the device structure. The scale bar is $100 \mathrm{~nm}$. (c) The current density-voltage characteristics of the perovskite solar cells on c-ITO/AgNW-GFRHybrimer, on c-ITO/CuNW-GFRHybrimer, on ITO/glass and on ITO/PET under $100 \mathrm{~mW} \mathrm{~cm}^{-2} \mathrm{AM} 1.5 \mathrm{G}$ illumination. Inset: a photograph of the flexible perovskite cell on the c-ITO/AgNW-GFRHybrimer film. (d) The normalized PCEs of the flexible perovskite devices on the c-ITO/metal NW-GFRHybrimer films and on the ITO/PET film as a function of bending cycle with different bending radii of 5 and $2.5 \mathrm{~mm}$. AgNW, silver nanowire; c-ITO, crystalline indium tin oxide; CuNW, copper nanowire; NW, nanowire; PCE, power conversion efficiency; PET, polyethylene terephthalate.

Table 2 Photovoltaic performance of the perovskite solar cells on c-ITO/AgNW-GFRHybrimer, c-ITO/CuNW-GFRHybrimer, ITO/glass and ITO/PET under $100 \mathrm{~mW} \mathrm{~cm}^{-2} \mathrm{AM} 1.5 \mathrm{G}$ illumination

\begin{tabular}{lcccc}
\hline Device & $\mathrm{V}_{o c}(\mathrm{~V})$ & $\mathrm{J}_{S C}\left(\mathrm{mAcm} \mathrm{cm}^{-2}\right)$ & $F F$ & PCE (\%) \\
\hline c-ITO/AgNW-GFRHybrimer & 0.99 & 21.53 & 0.66 & 14.15 \\
c-ITO/CuNW-GFRHybrimer & 0.97 & 20.09 & 0.66 & 12.95 \\
ITO/glass & 0.97 & 21.82 & 0.73 & 15.38 \\
ITO/PET & 0.96 & 20.50 & 0.62 & 12.08 \\
\hline
\end{tabular}

Abbreviations: AgNW, silver nanowire; c-ITO, crystalline indium tin oxide; CuNW, copper nanowire; PCE, power conversion efficiency; PET, polyethylene terephthalate.

were maintained at $\sim 90 \%$ and $80 \%$ of the initial PCE, respectively (Figure $4 \mathrm{~d}$ and Supplementary Figure S10). In sharp contrast, the devices on the ITO/PET film sharply deteriorated after 100 cycles of bending. To investigate the origin of the performance degradation of the devices, a surface scanning electron microscope analysis was conducted after the bending test (Supplementary Figure S11). For the reference device, cracks appeared because of the brittleness of the bottom ITO electrode. In contrast, the devices on the c-ITO/AgNWand c-ITO/CuNW-GFRHybrimer films exhibit no cracks after bending. It should be noted that the excellent flexibility of the perovskite solar cells on the c-ITO/metal NW-GFRHybrimer films is attributed to the outstanding mechanical stability of the c-ITO/metal NWGFRHybrimer films. ${ }^{13}$

\section{CONCLUSION}

In summary, we fabricated a flexible, thermally/chemically robust and high-performance c-ITO/metal NW hybrid TCE/substrate platform (c-ITO/metal NW-GFRHybrimer film). We examined the optoelectrical properties and the mechanical, chemical and thermal stabilities of the c-ITO/metal NW-GFRHybrimers with various ITO thicknesses. From an optoelectrical perspective, c-ITO can be beneficial for fabricating high-performance metal oxide/metal NW TCEs. The $10 \mathrm{~nm}$ thickness of c-ITO was found to be optimal, resulting in an outstanding flexibility of the metal NW TCEs, as well as excellent thermal/chemical stability. Perovskite solar cells were fabricated on the chemically stable c-ITO/metal NW-GFRHybrimer films, and the devices show excellent photovoltaic performance and outstanding mechanical durability. Our c-ITO/metal NW-GFRHybrimer films can be a robust TCE/substrate platform for various flexible optoelectronic devices.

\section{CONFLICT OF INTEREST}

The authors declare no conflict of interest.

\section{ACKNOWLEDGEMENTS}

This research was supported by a National Research Foundation of Korea (NRF) grant funded by the Korean government (MSIP) (CAFDC 5-3, NRF-2007-0056090) and the Graphene Materials and Components Development Program of MOTIE/KEIT (10044412). 
Author contributions: H-G Im and S Jeong conceived and designed the experiments and prepared the manuscript. J Jin, J Lee, D-Y Youn, W-T Koo, S-B Kang, H-J Kim, J Jang and D Lee prepared and characterized materials of the hybrid transparent electrodes. All authors discussed the results and commented on the manuscript.

1 Ellmer, K. Past achievements and future challenges in the development of optically transparent electrodes. Nat. Photon. 6, 809-817 (2012).

2 Gaynor, W., Burkhard, G. F., McGehee, M. D. \& Peumans, P. Smooth nanowire/polymer composite transparent electrodes. Adv. Mater. 23, 2905-2910 (2011).

3 An, B. W., Hyun, B. G., Kim, S. Y., Kim, M., Lee, M. S., Lee, K., Koo, J. B., Chu, H. Y., Bae, B. S. \& Park, J. U. Stretchable and transparent electrodes using hybrid structures of graphene-metal nanotrough networks with high performances and ultimate uniformity. Nano Lett. 14, 6322-6328 (2014).

4 Kim, K., Kim, J., Hyun, B. G., Ji, S., Kim, S. Y., Kim, S., An, B. W. \& Park, J. U. Stretchable and transparent electrodes based on in-plane structures. Nanoscale 7 14577 (2015).

5 An, B. W., Gwak, E. J., Kim, K., Kim, Y. C., Jang, J., Kim, J. Y. \& Park, J. U. Stretchable, transparent electrodes as wearable heaters using nanotrough networks of metallic glasses with superior mechanical properties and thermal stability. Nano Lett. 16, 471-478 (2016).

6 Hecht, D. S., Hu, L. \& Irvin, G. Emerging transparent electrodes based on thin films of carbon nanotubes, graphene, and metallic nanostructures. Adv. Mater. 23. 1482-1513 (2011)

7 Chen, Z., Li, W., Li, R., Zhang, Y., Xu, G. \& Cheng, H. Fabrication of highly transparent and conductive indium-tin oxide thin films with a high figure of merit via solution processing. Langmuir 29, 13836-13842 (2013).

8 Choi, M.-C., Kim, Y. \& Ha, C.-S. Polymers for flexible displays: from material selection to device applications. Prog. Polym. Sci. 33, 581-630 (2008).

9 Leterrier, Y., Médico, L., Demarco, F., Månson, J. A. E., Betz, U., Escolà, M. F., Kharrazi Olsson, M. \& Atamny, F. Mechanical integrity of transparent conductive oxide films for flexible polymer-based displays. Thin Solid Films 460, 156-166 (2004).

10 Kang, S.-B., Kim, H.-J., Noh, Y.-J., Na, S.-I. \& Kim, H.-K. Face-to-face transferred multicrystalline ITO films on colorless polyimide substrates for flexible organic solar cells. Nano Energy 11, 179-188 (2015).

11 Morikawa, H. \& Fujita, M. Crystallization and electrical property change on the annealing of amorphous indium-oxide and indium-tin-oxide thin films. Thin Solid Films 359, 61-67 (2000).

12 Bender, M., Trube, J. \& Stollenwerk, J. Deposition of transparent and conducting indium-tin-oxide films by the R.F.-superimposed dc sputtering technology. Thin Solid Films 354, 100-105 (1999).

13 Kim, B. J., Kim, D. H., Lee, Y.-Y., Shin, H.-W., Han, G. S., Hong, J. S., Mahmood, K., Ahn, T. K., Joo, Y.-C., Hong, K. S., Park, N.-G., Lee, S. \& Jung, H. S. Highly efficient and bending durable perovskite solar cells: Toward a wearable power source. Energy Environ. Sci. 8, 916-921 (2015).

14 Gaynor, W., Hofmann, S., Christoforo, M. G., Sachse, C., Mehra, S., Salleo, A., McGehee, M. D., Gather, M. C., Lüssem, B., Müller-Meskamp, L., Peumans, P. \& Leo, K. Color in the corners: ITO-free white oleds with angular color stability. Adv. Mater. 25, 4006-4013 (2013).

15 Hu, L., Kim, H. S., Lee, J. Y., Peumans, P. \& Cui, Y. Scalable coating and properties of transparent, flexible, silver nanowire electrodes. ACS Nano 4, 2955-2963 (2010).

16 Lee, J., Lee, P., Lee, H. B., Hong, S., Lee, I., Yeo, J., Lee, S. S., Kim, T. S., Lee, D. \& Ko, S. H. Room-temperature nanosoldering of a very long metal nanowire network by conducting-polymer-assisted joining for a flexible touch-panel application. Adv. Funct. Mater. 23, 4171-4176 (2013).

17 Im, H. G., Jung, S. H., Jin, J., Lee, D., Lee, J., Lee, D., Lee, J. Y., Kim, I. D. \& Bae, B. S. Flexible transparent conducting hybrid film using a surface-embedded copper nanowire network: a highly oxidation-resistant copper nanowire electrode for flexible optoelectronics. ACS Nano 8, 10973-10979 (2014).

18 Lee, M. S., Lee, K., Kim, S. Y., Lee, H., Park, J., Choi, K. H., Kim, H. K., Kim, D. G., Lee, D. Y., Nam, S. \& Park, J. U. High-performance, transparent, and stretchable electrodes using graphene-metal nanowire hybrid structures. Nano Lett. 13, 2814-2821 (2013)

19 Lee, J. Y., Connor, S. T., Cui, Y. \& Peumans, P. Solution-processed metal nanowire mesh transparent electrodes. Nano Lett. 8, 689-692 (2008).

20 Leem, D. S., Edwards, A., Faist, M., Nelson, J., Bradley, D. D. \& de Mello, J. C. Efficient organic solar cells with solution-processed silver nanowire electrodes. Adv. Mater. 23, 4371-4375 (2011)

21 Liang, J., Li, L., Niu, X., Yu, Z. \& Pei, Q. Elastomeric polymer light-emitting devices and displays. Nat. Photon 7, 817-824 (2013).

22 Jin, J., Lee, J., Jeong, S., Yang, S., Ko, J.-H., Im, H.-G., Baek, S.-W., Lee, J.-Y. \& Bae, B.-S. High-performance hybrid plastic films: A robust electrode platform for thin-film optoelectronics. Energy Environ. Sci. 6, 1811-1817 (2013).
23 Sachse, C., Weiß, N., Gaponik, N., Müller-Meskamp, L., Eychmüller, A. \& Leo, K. ITOfree, small-molecule organic solar cells on spray-coated copper-nanowire-based transparent electrodes. Adv. Energy Mater. 4, 1300737 (2014).

$24 \mathrm{Im}$, H. G., Jin, J., Ko, J. H., Lee, J., Lee, J. Y. \& Bae, B. S. Flexible transparent conducting composite films using a monolithically embedded AgNW electrode with robust performance stability. Nanoscale 6, 711-715 (2013).

25 Lee, D., Lee, H., Ahn, Y., Jeong, Y., Lee, D. Y. \& Lee, Y. Highly stable and flexible silver nanowire-graphene hybrid transparent conducting electrodes for emerging optoelectronic devices. Nanoscale 5, 7750-7755 (2013).

26 Won, Y., Kim, A., Lee, D., Yang, W., Woo, K., Jeong, S. \& Moon, J. Annealing-free fabrication of highly oxidation-resistive copper nanowire composite conductors for photovoltaics. NPG Asia Mater. 6, e105 (2014).

27 Chen, Z., Ye, S., Stewart, I. E. \& Wiley, B. J. Copper nanowire networks with transparent oxide shells that prevent oxidation without reducing transmittance. ACS Nano 8 , 9673-9679 (2014)

28 Han, J., Yuan, S., Liu, L., Quu, X., Gong, H., Yang, X., Li, C., Hao, Y. \& Cao, B. Fully indium-free flexible Ag nanowires/ZnO:F composite transparent conductive electrodes with high haze. J. Mater. Chem. A 3, 5375-5384 (2015).

29 Göbelt, M., Keding, R., Schmitt, S. W., Hoffmann, B., Jäckle, S., Latzel, M., Radmilović, V. V., Radmilović, V. R., Spiecker, E. \& Christiansen, S. Encapsulation of silver nanowire networks by atomic layer deposition for indium-free transparent electrodes. Nano Energy 16, 196-206 (2015).

30 Huang, Q., Shen, W., Fang, X., Chen, G., Yang, Y., Huang, J., Tan, R. \& Song, W. Highly thermostable, flexible, transparent, and conductive films on polyimide substrate with an AZO/AgNW/AZO structure. ACS Appl. Mater. Interfaces 7, 4299-4305 (2015).

31 Jin, J., Ko, J. H., Yang, S. \& Bae, B. S. Rollable transparent glass-fabric reinforced composite substrate for flexible devices. Adv. Mater. 22, 4510-4515 (2010).

32 Elechiguerra, J. L., Larios-Lopez, L., Liu, C., Garcia-Gutierrez, D., Camacho-Bragado, A. \& Yacaman, M. J. Corrosion at the nanoscale: the case of silver nanowires and nanoparticles. Chem. Mater. 17, 6042-6052 (2005).

33 Chang, Y., Lye, M. L. \& Zeng, H. C. Large-scale synthesis of high-quality ultralong copper nanowires. Langmuir 21, 3746-3748 (2005).

34 Tuna, O., Selamet, Y., Aygun, G. \& Ozyuzer, L. High quality ITO thin films grown by dc and rf sputtering without oxygen. J. Phys. D Appl. Phys. 43, 055402 (2010).

35 De, S., King, P. J., Lyons, P. E., Khan, U. \& Coleman, J. N. Size effects and the problem with percolation in nanostructured transparent conductors. ACS Nano 4, 7064-7072 (2010).

36 Lee, H. J., Hwang, J. H., Choi, K. B., Jung, S. G., Kim, K. N., Shim, Y. S., Park, C. H., Park, Y. W. \& Ju, B. K. Effective indium-doped zinc oxide buffer layer on silver nanowires for electrically highly stable, flexible, transparent, and conductive composite electrodes. ACS Appl. Mater. Interfaces 5, 10397-10403 (2013).

$37 \mathrm{Kim}, \mathrm{A}$., Won, Y., Woo, K., Jeong, S. \& Moon, J. All-solution-processed indium-free transparent composite electrodes based on $\mathrm{Ag}$ nanowire and metal oxide for thin-film solar cells. Adv. Funct. Mater. 24, 2462-2471 (2014).

38 Kholmanov, I. N., Domingues, S. H., Chou, H., Wang, X., Tan, C., Kim, J. Y., Li, H., Piner, R., Zarbin, A. J. \& Ruoff, R. S. Reduced graphene oxide/copper nanowire hybrid films as high-performance transparent electrodes. ACS Nano 7, 1811-1816 (2013).

$39 \mathrm{Kim}, \mathrm{A}$. Won, Y., Woo, K. Kim, C.-H. \& Moon, J. Highly transparent low resistance $\mathrm{ZnO} / \mathrm{Ag}$ nanowire/ZnO composite electrode for thin film solar cells. ACS Nano 7, 1080-1091 (2013)

40 Della Gaspera, E., Peng, Y., Hou, Q., Spiccia, L., Bach, U., Jasieniak, J. J. \& Cheng, Y.-B. Ultra-thin high efficiency semitransparent perovskite solar cells. Nano Energy 13, 249-257 (2015).

41 Robertson, J. Band alignment at metal-semiconductor and metal-oxide interfaces. Phys. Status Solidi A 207, 261-269 (2010).

42 Kim, Y., Ryu, T. I., Ok, K.-H., Kwak, M.-G., Park, S., Park, N.-G., Han, C. J., Kim, B. S., Ko, M. J., Son, H. J. \& Kim, J.-W. Inverted layer-by-layer fabrication of an ultraflexible and transparent $\mathrm{Ag}$ nanowire/conductive polymer composite electrode for use in highperformance organic solar cells. Adv. Funct. Mater. 25, 4580-4589 (2015).

43 Kim, Y. H., Müller-Meskamp, L. \& Leo, K. Ultratransparent polymer/semitransparent silver grid hybrid electrodes for small-molecule organic solar cells. Adv. Energy Mater. 5, 1401822 (2015).

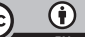

This work is licensed under a Creative Commons Attribution 4.0 International License. The images or other third party material in this article are included in the article's Creative Commons license, unless indicated otherwise in the credit line; if the material is not included under the Creative Commons license, users will need to obtain permission from the license holder to reproduce the material. To view a copy of this license, visit http:// creativecommons.org/licenses/by/4.0/

(C) The Author(s) 2016

Supplementary Information accompanies the paper on the NPG Asia Materials website (http://www.nature.com/am) 